\title{
A Segmentation Method of Fused Multispectral and Panchromatic Images using Quick Shift Algorithm and Brovey Transform
}

\author{
Vanmathi C
}

\begin{abstract}
Satellite Image Processing has always been a good domain to carry out researches. This is mainly due to the probability of the vast nature of the domain. There are many Image fusions and segmentation methods are available in the area of Satellite Image Processing. The presented research work proposes the image fusion method to inter twined the high determination panchromatic (PAN) picture and the low-determination multispectral picture to gain the multispectral image of high determination. For picture fusion the Brovey transform is used. The derived inter twined picture is granted as an input to the further segmentation process. Segmentation of the image obtained after the fusion is done and the recognition of land, marine, plantation and residential ranges are shown in the picture of the particular location gained from the satellite.

For the segmentation of the fused satellite picture the Quick Shift Segmentation clustering technique is used. Utilizing Quick Shift Segmentation on the combined image the various sections can easily be identified as compared to the unprocessed picture gain from the satellite. Scientists utilize Brovey transform to intertwined $R G B$ colour pictures to high determination panchromatic (PAN) pictures. In the proposed work, the main focus is to degrade spectral irregularity and enhancement of spatial data on merging the pictures of satellite Limitation of the current technique of the combination of images for example restricted directionality, shift invariant, absence of the data are amended by using Brovey transformation. The outcomes of the current system are generally unsatisfied. To overcome this Quick Shift Segmentation technique is used with the Brovey transform in the proposed system. The exactness of segmented image is checked with respect to reference information. The fused image segmentation outcomes have been ascertained and it shows that Brovey transform with interaction of Quick Shift Segmentation technique provides more successive outcomes.
\end{abstract}

Keywords : Brovey transform, Quick Shift Segmentation technique, Satellite Image Processing, Panchromatic and MS images.

\section{INTRODUCTION}

\section{A. General}

Remote sensing is the study of fetching information about the land surface without being entirely contact. Several satellites are present to acquire the satellite picture of any segment of the land surface [1]. The characteristics of the images are varying according to the satellite which they are

Revised Manuscript Received on December 20, 2019.

* Correspondence Author

Vanmathi C*, School of Information Technology and Engineering, Vellore Institute of Technology, Vellore, India, Email: Vanmathi.c@vit.ac.in taken from. The images captured from the satellite are having diverse effect some of the pictures having less determination and some are having high determination. Contingent on the applications the images are used by the specialist.

Remote detection of the land surface offers a tremendous variation of image information [2], in which the images have different characteristics like temporal, radiometric, spatial and spectral resolutions. For the digital satellite sensing, imaging system offers an exchange-off between the changed variations of the images and their determination quality. Some provides high spatial and some provides high ghastly determination. There is no such a framework that offers both. Picture from the satellite are utilized for the prevision of environment and the identification of particular region. For some applications, there is a need to distinguish among the section of the land portion which satellite image are been utilized. It is found that raw satellite picture is unable to provide the accurate information of the section of the land for example, water, cultivation area, soil and developed living section from the satellite picture which has been taken. The successful identification of the particular region will help the researches to effectively analyse and monitor. For the detection and analysis of these areas the segmentation and clustering is done using Quick Shift Segmentation technique. Using these techniques it becomes easy to detect the environment and the particular area. Remote sensing picture combination has various application domains. A principal domain is the multi resolution intertwined picture usually known as Pan-sharpening of satellite pictures. Pictures captured from satellite are mainly of two types: Multispectral and Panchromatic. A multispectral picture [3] is a picture that includes more than one shaded band. It is pliant by a sensor which is able to divide the illumination reflected from the ground in the form of discrete light bands. A multispectral picture is a shaded picture which contains three bands. Multispectral picture is a picture that includes more than one shaded band. It is pliant by a sensor which is able to divide the illumination reflected from the ground in the form of discrete light bands. A multispectral picture is a shaded picture which contains three bands consists of shaded bands usually red, blue and green in color having different wavelength in the electromagnetic spectrum. Multispectral pictures plotting are done to take privilege of the various illumination properties of ground surface elements [4]. 


\section{A Segmentation Method of Fused Multispectral and Panchromatic Images using Quick Shift Algorithm and Brovey Transform}

Multispectral pictures are used for recognition of land, marine, plantation, residential ranges and in remote sensing applications. Satellites generally take several pictures from different angles. A multispectral picture is a collection of several colored images of the same area, each of them acquired from different sensors. Limitation of multi-spectral pictures is that here we have to compute additional data, so the memory and processing time requirement will increase [5]. In opposition to multispectral picture, a panchromatic picture includes only one broad band of reflectance data. The data is generally an interpretation of a range of bands and wavelengths. Panchromatic picture usually contain higher spatial determination as compared to multispectral picture because the wide spectral range permits smaller detectors to be used while the management of high signal to noise ratio[6]. A panchromatic picture is usually represented in the form of gray level image. The represented illumination of a particular pixel of picture is relative to the pixel digital values which is related to the magnitude of radiation coming from sun reflected by the targets in the pixel and can be easily be sensed [7]. A panchromatic picture is displayed as a black and white picture taken from satellite of a particular area with narrow wavelength. Four major types of information available in the visual picture which is usually used for picture portrayal and examination are Radiometric Information illumination, intensity, tone, brightness, Statistical and Contextual Information, Spectral Information (hue, shade) and Surface Information.

\section{B. Motivation}

The real problem in satellite picture processing is that the raw satellite picture does not give the exact information of the sections for example, marine, cultivation part, land, industrial and residential section. Hence in this paper efforts are made to detect such sections effectively from the captured satellite pictures $[8,9,10]$. The fruitful identification of the particular section will encourage the researches to successfully analyse and screen. The satellite pictures contain some contortion which does not provides the exact outcome[11]. But the melded satellite picture removes this distorted picture since it takes at least two pictures and extracts the pertinent information of those pictures into a solitary picture [12]. In this paper, firstly satellite picture is intertwined and afterwards segmentation of the fused satellite picture has been performed.

\section{Our Approach}

In this research work, for the detection and investigation of the sections, for example, marine, land, cultivation section, and residential area are segmented and clustered using Quick Shift Segmentation strategy. Utilizing these techniques it becomes easy to recognize nature and the particular territory. But these satellite pictures have low spatial determination. Panchromatic picture is the picture with high determination. So in the given proposed strategy firstly we combine this high determination panchromatic picture with less determination multispectral picture to grab high determination multispectral picture and we also obtain the intensity hue saturated image. In this paper, Brovey transformation is utilized for the picture merging. These combined pictures are used for further segmentation utilizing Quick Shift Segmentation calculation. Picture fusion object is to combine the multiple pictures information into one picture, which is more suitable for the

image processing investigation. And Image segmentation is a process of dividing an image into different regions. Here for the easier identification of areas the areas are denoted by using different color.

\section{OVERVIEW OF PROPOSED WORK}

\section{A. Architecture of Proposed Work}

This section describes the overview of the techniques applied for the proposed system for Satellite image fusion and segmentation for finding the built-up area, water, Hill and vegetation area. The proposed method uses Brovey for image fusion with Quick Shift Segmentation Algorithm for segmentation and clustering is done. The architecture of the proposed algorithm is showed in the fig 2.1.

\section{- Image Pre-processing}

Pre-processing is a process of betterment of the picture data which detract involuntary mutilation and enhances the features of the picture required for further processing. Pre-processing of picture taken from satellite is the initial step in the process of image preparation [13]. Pre processing consists of a various operations which incorporate atmospheric adjustment, normalization, concealing and geometric transformations (rotation, scaling, and translation) [14]. Radiometric revision and geometric correction is the often done in picture pre-handling.

Radiometric redresses are performing to improve the brightness percentage, of the ground objects, that are mutilated because of sensor calibration or sensor malfunction causes [15].

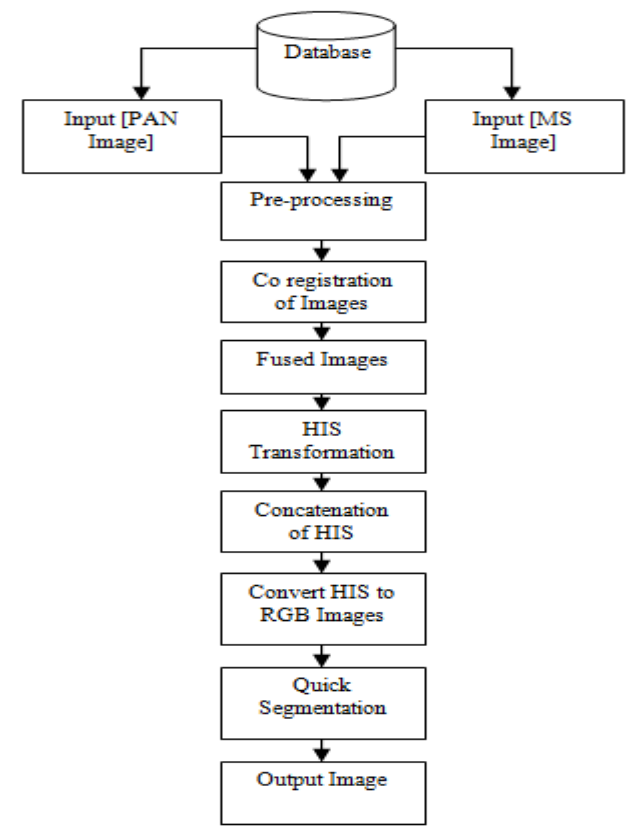

Fig. 1 Architecture for Proposed Method

Because of the disseminating of reflected electromagnetic light, the mutilation is brought on in the satellite pictures due to the changing climate. 
Geometric redresses are performing to rectify the inaccuracy between the pixels of the picture elements in the satellite picture data, and the genuine location of pixel on the ground. System, precision and terrain adjustments are the various sorts of geometric remedies [16]. Satellite Image data recorded by sensors contains some error cognate to geometrical and illumination factor of constituent of the image. Using appropriate mathematical models these errors are corrected. Image enhancement improves the pixel brightness values of the image. Noise filtering techniques are used to remove the various types of noise present in the captured image. Various filters like high pass, mean, median has applied.

\section{- Co-registration of pictures}

Image enrolment involves detection and matching of patterns from satellite pictures takes from different sensors, timing and viewpoints by manipulation and preparing in remotely detected data used for many applications[17]. During the process of image enrolment different arrangements of data is transformed into one coordinate framework. Pictures with different sizes, corner and magnitude are detected and expelled while testing for coordinating. Pictures acquired for satellite picture combination may be taken from different sensors, angles, clips and depths $[18,19]$. So it is necessary to alter diverse arrangements of data into one coordinate framework. Picture enrolment phase is used as a part of computer imaging, picture combination, clinical imaging, environment monitoring and target acknowledgement in military and satellite picture handling.

Picture registration implies accurate pixel-to-pixel coordinating of two different pictures. The following sorts of enrolment can be performed on the data acquired from multiple sources:

- Multimodal Registration

- Temporal Registration

- Angle Registration

- Model Registration

Satellite picture enlistment is an ambitious step in picture handling to resemble and adjust different images acquired from different climate conditions or by using diverse sensors or at different angles. Picture registration is mainly used in the area of remote detection. Due to the different class of satellite pictures, it is important to ad-here different systems to each registration work[20].

Images which are co-registered are free from the noises and the sizes of the image are fixed. Image co-registration is utilized for geometrically correcting the multiple pictures to grab fuse corresponding pixels that represents the same object. Image co-registration tool allows to automatic generation of tie points and rejects the irregular pictures, thus the automatic registration of image is possible.

\section{- Pan sharpening}

Pan sharpening is a procedure of consolidating high determination panchromatic picture and low determination multispectral picture to get a solitary high determination shaded picture. Pan sharpening of pictures is applied to sharpen the picture so that the fidelity of picture can be maintained. Summation of low determination colour image along with the high determination colour image provides the high determination multispectral shaded image[21].
Pan Sharpening is a short frame for "Panchromatic sharpening". To build the spatial determination of a multispectral picture, a panchromatic (single band) image is used. Panchromatic picture have low level of determination than multispectral picture, while frequently a multispectral picture will have a lower spatial determination than panchromatic picture. A pan sharpened picture displays a picture fusion of remote detecting satellite pictures: the multispectral and the panchromatic pictures which provide the optimum of both high phantom determination and high spatial determination [22].

The commonly used calculation for pan sharpening is known as "component substitution". That pertain the following steps:

- Up-sampling - as the similar determination of panchromatic band the other shading bands are up-sampled.

- Arrangement - the panchromatic band and the up-examined color bands are arranged to deflate surface due to reduction in picture enrolment process.

- Forward transform - the up-sampled shaded bands are changes into an alternate shade space.

- Magnitude matching - the magnitude of the color bands is duplicated to the pan band intensity in the changed space.

- Component replacement - the pan band is then straight replaced into intensity part.

- Reverse change - the reverse change is done using the substituted intensity part to change back to the original shaded space.

\section{Picture Fusion Method}

Multi sensor picture fusion is the process toward consolidating useful information of at least two pictures into a solitary picture [23] . The resulting picture provides more information than the given injected pictures. In the field of combination of satellite picture many algorithms are available. For the exact portrayal of the earth surface the satellite picture should contain high spatial and high ghostly determination in a single picture. The raw satellite picture will not contain both high spatial and high phantom determination in one single picture that is the reason image combination is the fundamental step in process of satellite picture processing. Panchromatic pictures are the image obtained from wide wavelength range and it is displayed in black and white. Panchromatic picture is obtained by the satellite which goes with greatest determination. Multispectral pictures are the shade image contains three shaded bands namely red, green and blue. These pictures are acquired in more than one spectral band on different time slice. A solitary image of the same territory contains distinct spectral bands [24].

\section{- Transformation using Brovey Method}

The transformation using Brovey is utilized to blend the PAN and MS pictures. Every MS picture is duplicated by a proportion of the pixel value of a picture isolated by the entirely of the MS pictures. Combined RGB shaded bands containing picture is characterized by the following condition, the R,G and B components of MS pictures is multiplied with the PAN pictures. 


\section{A Segmentation Method of Fused Multispectral and Panchromatic Images using Quick Shift Algorithm and Brovey Transform}

Many Scientists were make use of the BT method to intertwine RGB shaded MS pictures with the high determination PAN pictures. BT is restricted to three bands and the multiplicative system provides radiometric contortion. Experienced examiner is required for using this system to grab the fruitful outcomes. It avoids the advancement of user-friendly framework. Shading effects can be degrading sometimes by using BT method [25].

- The BT is constrained to three shaded bands and multiplicative procedures present a radiometric mutilation.

- Computational request is less and Less time consuming

- Spatial details are secured in this method.

- Easy to process

- Used as a benchmark for analysis of other fusion strategies.

- High variations pixel values in data.

- Pictures are discouraged in value of the intertwined picture.

- BT strategy does not offer any guarantee for the exactness of the fused pictures.

\section{- RGB to HSI Transformation}

In the RGB color model each band is represented by Red, Green and Blue color. The colours are derived using three parametric values ranging from 0 to 255 . The HSI model encodes the color in three parameters where $\mathrm{H}$ denotes Hue, $\mathrm{S}$ denotes Saturation and I denote Intensity of the Image [26]. The picture can be combined by transformations based on Red-Green-Blue (RGB) to Intensity -Hue- Saturation (IHS).The following procedures are involve in this conversion:

- The less determination MS pictures can be re-sized as same to the PAN pictures.

- Change the R, G, B shaded bands of the multispectral image into the IHS components.

- Alter the panchromatic picture on the basis of multispectral picture by using the histogram matching and get the intensity component of the multispectral pictures.

- Replace the intensity component of MS by the PAN picture and then do inverse transformation to retrieve a high determination MS picture.

Steps for conversion

1. Read a RGB image

2. Represent the RGB image in the range $[0,1]$

3. Calculate HSI components

Intensity can be calculated by taking the average of the three shaded bands.

$\mathrm{I}=$ Average $(\mathrm{R}, \mathrm{G}, \mathrm{B})$

\section{- HSI to RGB Transformation}

After the retrieval of the intensity component from the HSI picture the Intensity graph has been drawn and the HSI picture is again converted into a shaded RGB picture for displaying the outcome of a system. This is a shaded RGB segmented picture by the use of which detection of the particular section of the land part becomes easier. The shaded bands of RGB are obtained by calculating the intensity values with hue and saturation points.

- Quick Shift Segmentation Algorithm

Quick shift is a quick mode looking for calculation like the average move. Quick shift calculation segments a RGB picture or any format picture according to the shade and brightness of band. It distinguishes group of pixels whose branches are labelled with separation esteem [27]. From this the progressive division of the picture has been indicated with sections relating to sub graphs. Valuable super pixels can be analyzed by separating the branches whose separation label is more than the given limit. (Limit can either be fixed manually or dictated by cross approval). After the Segmentation of fused satellite image we can easily identify the cultivation, land, marine and living sections [28]. Each section is represented by different color to which makes the better identification of the areas.

\section{B. Advantages of this architecture}

1. It helps to determination of soil, marine, cultivation land, industrial and residential area etc on the captured image.

2. It provides better quality of intertwined image with higher spatial and better spectral features.

3. By using the proposed image fusion method the distortion has been removed from the input satellite image.

4. It provides faster result then the current systems.

5. It provides shaded segmentation of thee output image, which makes it better for visualization of any object or to trade an object in a particular location.

\section{Proposed Work}

Static view: shows the static structure of the framework using elements, attributes and their relationships. Class diagrams and composite diagrams are come under this category.

Dynamic view: shows the dynamic behavior of the system by showing the interaction of the system among entities between the internal states of objects. Sequence diagrams, activity diagram and state diagrams are included under this category.

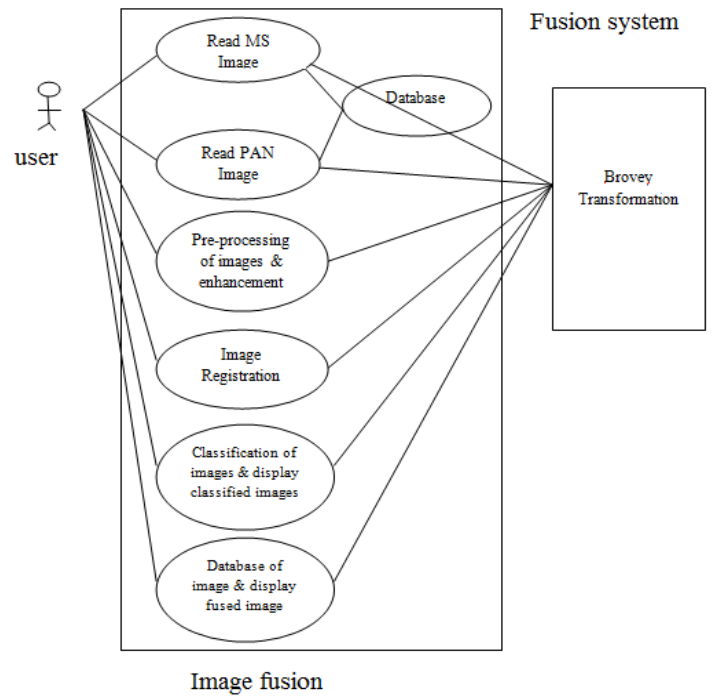

Fig. 2 Use Case for Image Fusion 


\section{EXPERIMENTAL RESULTS}

In this section the proposed method is evaluated on available satellite image dataset. This dataset contains the satellite image which contains panchromatic images and multispectral images. The trained PAN image and MS image is given as an input a retrieved an intertwined picture. Co-sized multispectral picture will be obtained initially then the Intensity Hue Saturated image will get by using the Brovey Algorithm [29]. And by using Quick segmentation technique the particular areas has been segmented and it is represented by using different colours for each type of area. Segmentation is performed in the fused satellite picture to retrieve marine, land, cultivation and residential sections.

A panchromatic image (Fig. 3) consists of only one band. It is usually represented as a black and white image or a greyscale image. The radiometric information is taken into considerations [30]. Panchromatic images usually contain higher spatial resolution than a multispectral image because the wide spectral range permits smaller detectors to be used while the management of high signal to noise ratio. Multispectral Image (Fig. 3.2) is a combination of many single image of the particular location obtains from different sensors. Multispectral image contains of three colored band which is a RGB color band where R stands for Red, G stands for Green and B stands for Blue. Each of this color bands is of different wavelengths. Multispectral images are most often used for remote sensing applications. But for processing extra data the multispectral image processing time and memory will require more and the speed will increase but the range of memory will decrease.

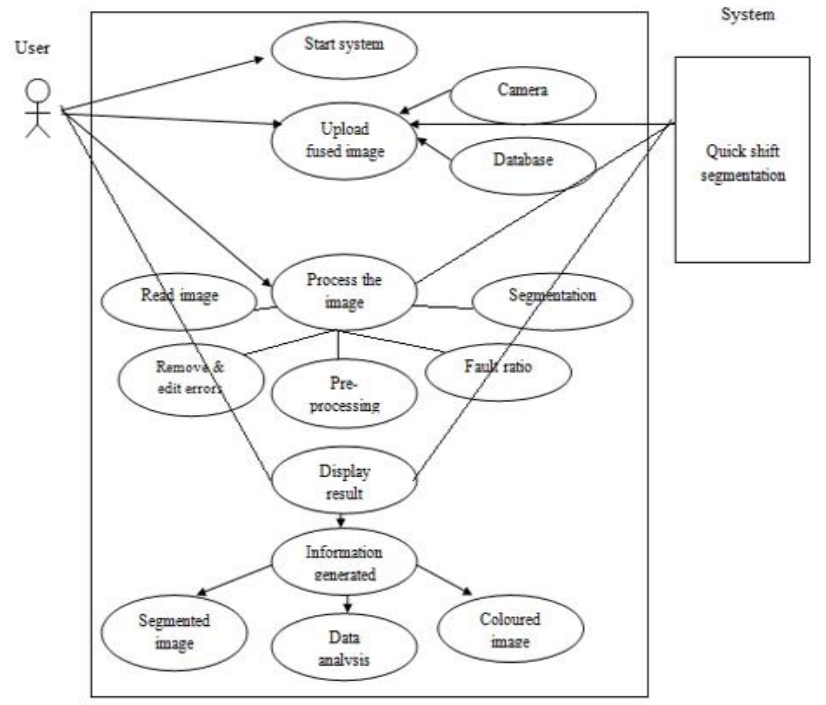

Fig. 3 Use Case of Image Segmentation

Re-sizing the Multispectral image according to the preferred input image size so that it can be forward for further processing. The Multispectral image can be re-sized based on the scaling value of panchromatic image. Here we are taken the scaling of images as $3 \times 3$.

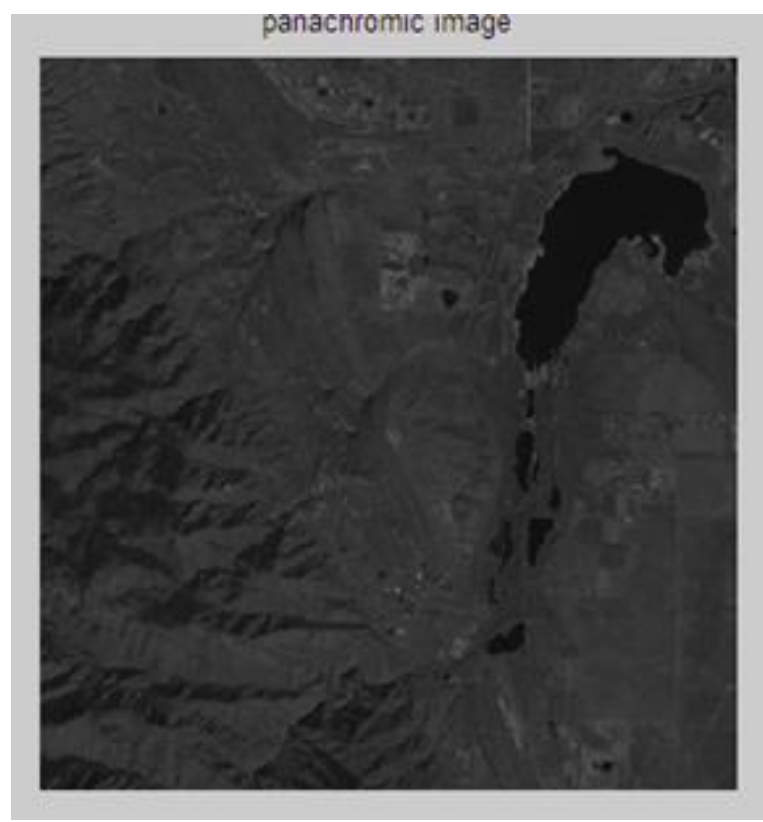

Fig. 4 Input PAN Image

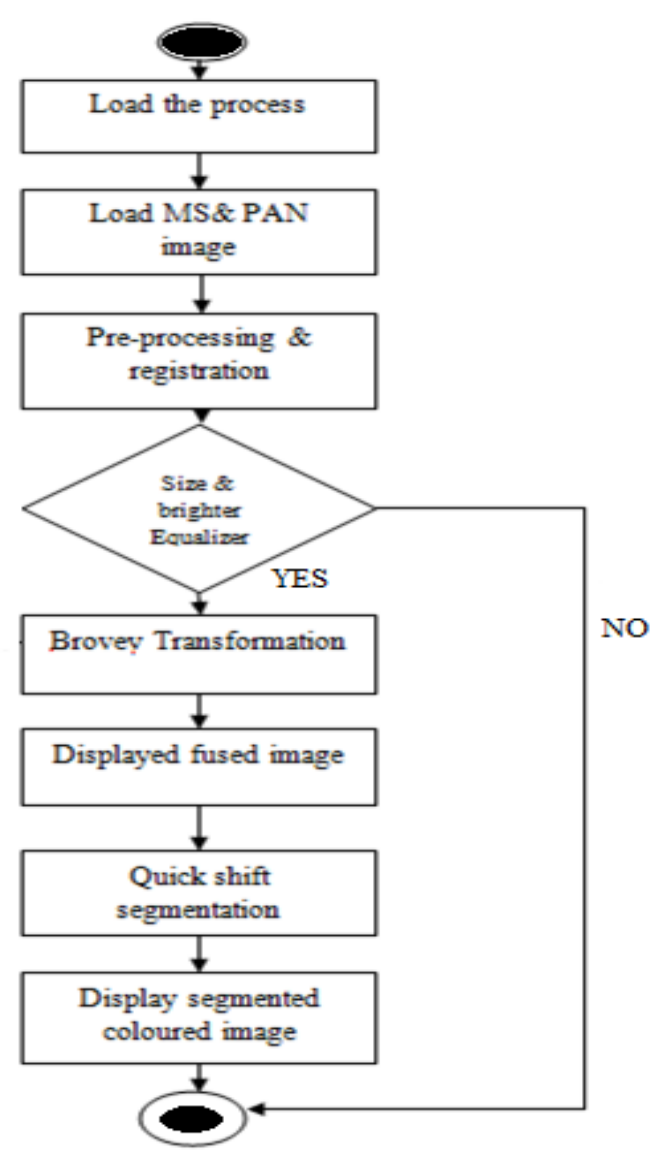

Fig. 5 Flow diagram of Proposed Method 


\section{A Segmentation Method of Fused Multispectral and Panchromatic Images using Quick Shift Algorithm}

and Brovey Transform

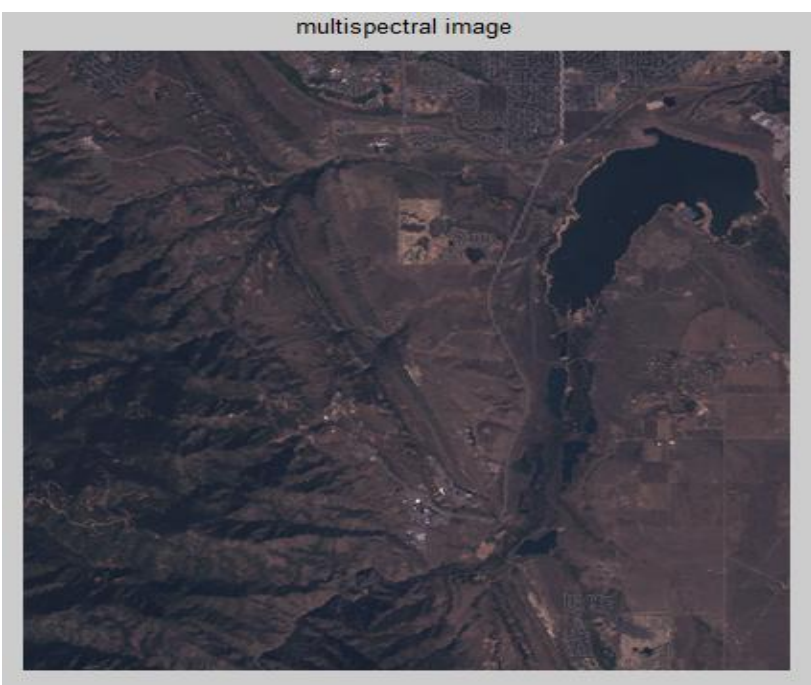

Fig. 6 Input MS Image

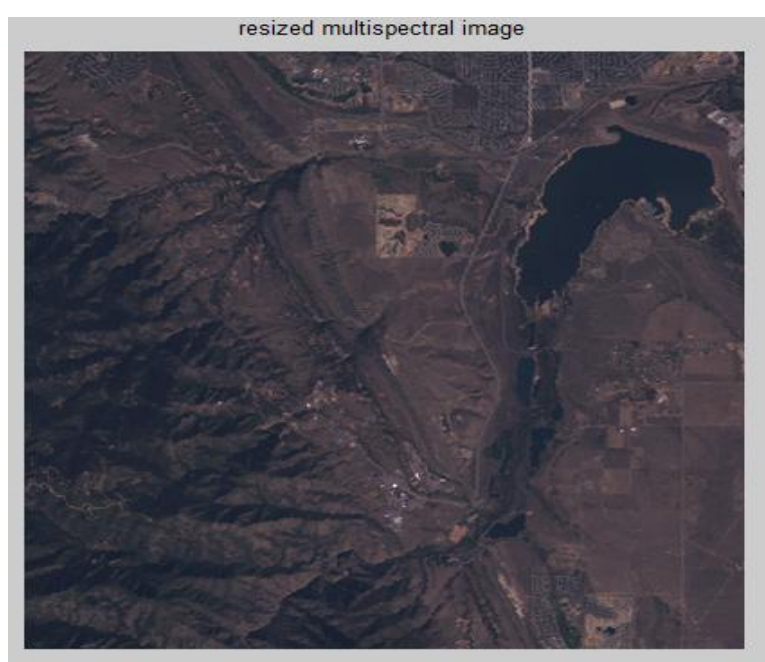

Fig. 7 Resized MS Image

Image registration is a process of changing different values of data into one common coordinate system. The images in the remote sensing applications are taken from different sources are stored in the dataset. The images in the dataset are taken in different time span, depth and by different angels are integrate to obtain the further result and it is co-registered the image which contains the error values are removed from this stage.

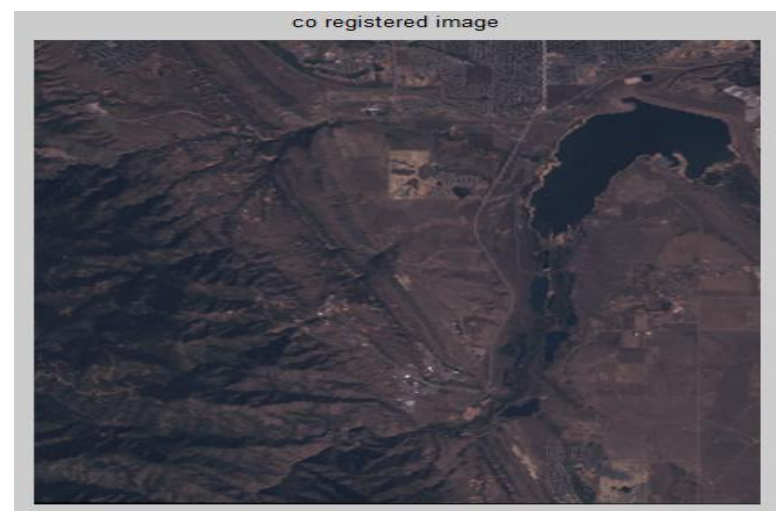

Fig. 8 Co-registered Image

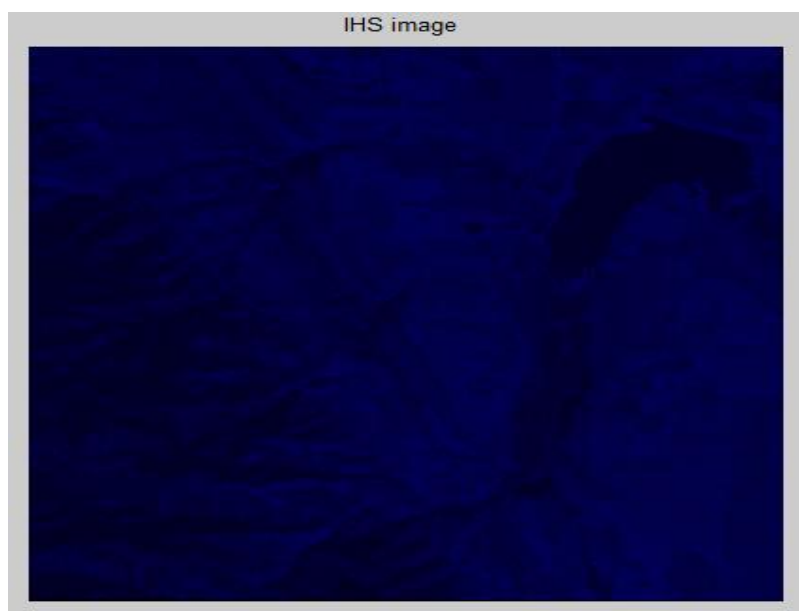

Fig. 9 IHS Image

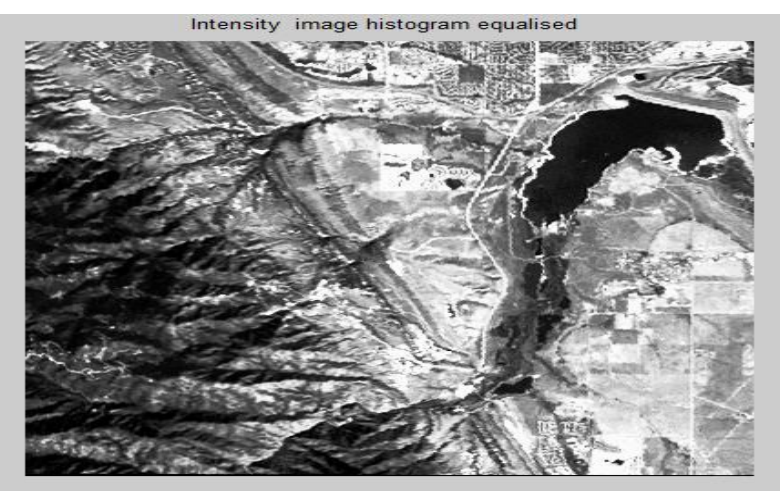

Fig. 10 Intensity Image

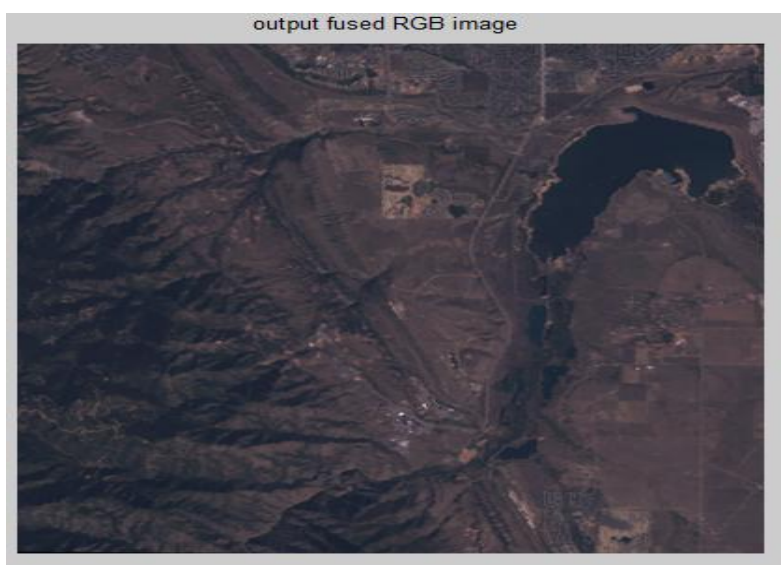

Fig. 11 Fused Image

The Intensity- Hue- Saturated image is a image which deals with the brightness of the image. Here the RGB color model is transformed into IHS model to obtain the intensity component from it. IHS is the common model used for the computer vision applications

Intensity image is taken from the IHS image it only considers the intensity component from the image and further the IHS image is again converted into RGB colored image.

The both multispectral and panchromatic images are fused to obtain the image of better quality which contains more useful information than the raw satellite image. The resultant image contains a high spectral and spatial resolution multispectral image. This Multispectral image is used further for the segmentation process.

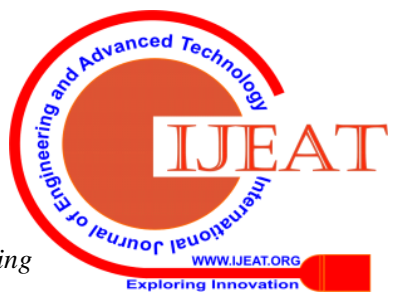


The fused image is used as a input for the image segmentation process the image is segmented based on the region of interest and their particular features. The satellite images for the land surface is segmented based on the particular areas such as land, vegetation, river and industrial area. The segmentation is performed by using Quick -Shift segmentation techniques.

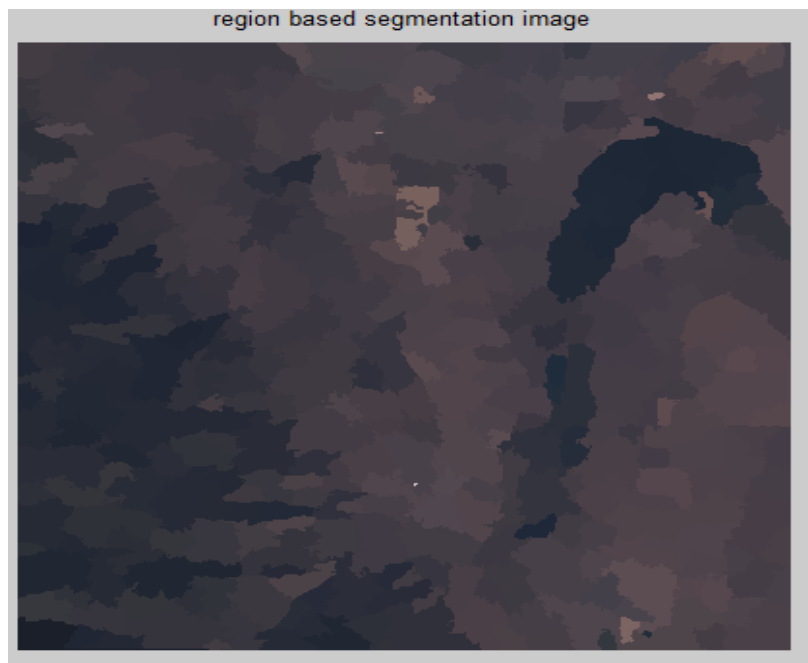

Fig. 12 Region based Segmented Image

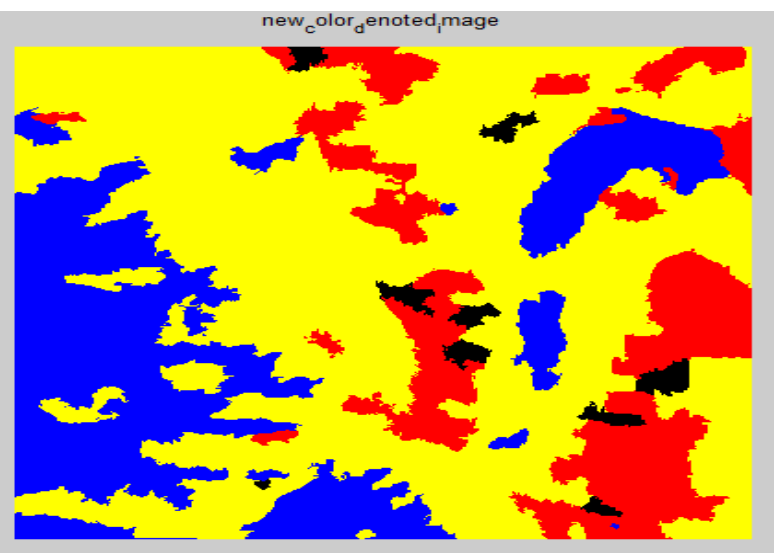

Fig. 13 Segmented Image

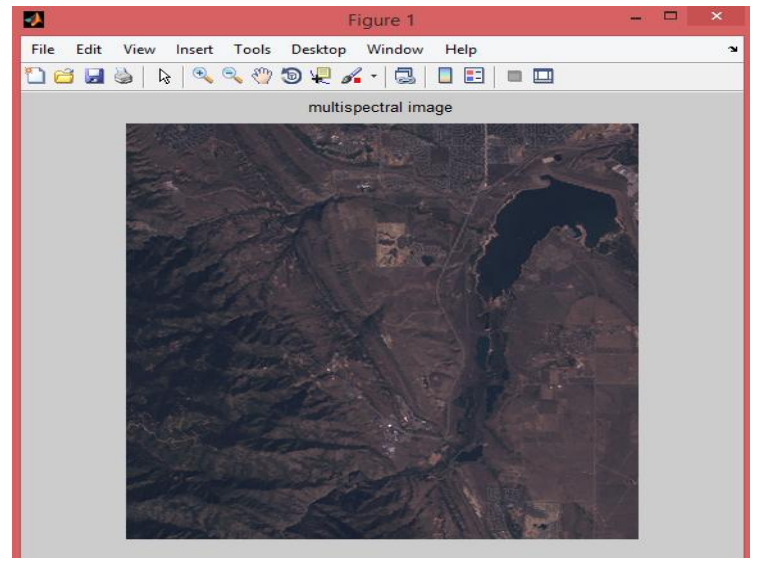

Fig. 14 Input Image

The color segmentation of the segmented image makes it easier for the researcher to detect a particular object or track a particular object in the given location. Due to the use of color it can be easily identified because finding the location is easy in the color segmentation technique.

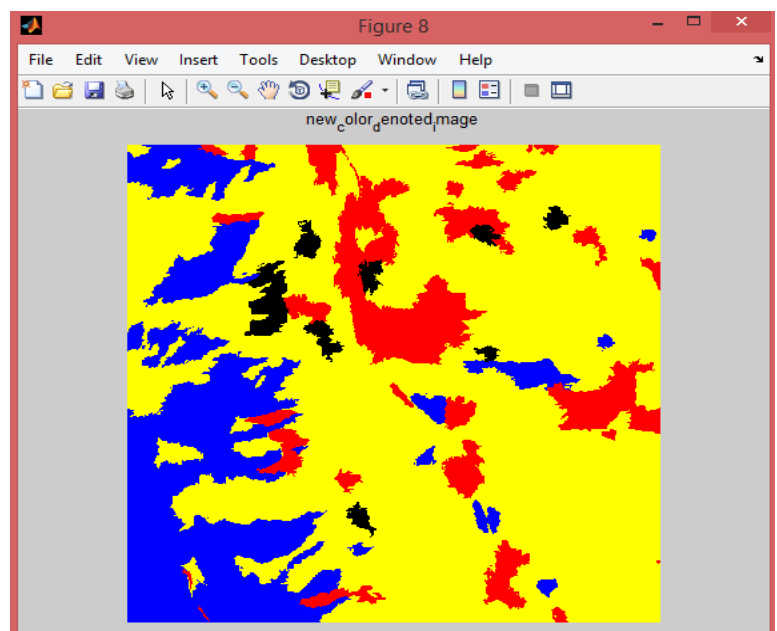

Fig. 15 Output Segmented Image

\section{CONCLUSION AND FUTURE WORK}

In this work, image fusion method has been proposed to retrieve high-determination multispectral images from a high-determination panchromatic (PAN) picture and less determination multispectral (MS) image. In the fused satellite image segmentation is performed by using Quick Shift Segmentation to segment and identify the marine, residential, cultivation land and industrial sections present in the region of the captured satellite image. And the regions are mentioned in different colours so that it can be easily identified.

Future research work can be extended to track a particular object at a particular location according to the provided shaded space. Picture fusion ways has been developed to take data from multiple sensors and to provide high determination picture for processing to the users. Enhance the rapid response capability for providing high determination information.

\section{REFERENCES}

1. Shingare, Pratibha P., Priya M. Hemane, and Duhita S. Dandekar. "Regression tree algorithm for classification of fused multispectral and panchromatic image." Advances in Computing, Communications and Informatics (ICACCI, 2014 International Conference on. IEEE, 2014.

2. Mandhare, Rohan Ashok, Pragati Upadhyay, and Sudha Gupta. "Pixel-level image fusion using brovey transforme and wavelet transform." International Journal of Advanced Research in Electrical, Electronics and Instrumentation Engineering 2.6 (2013): 2690-2695.

3. Kumar, N. S., \& Arun, M. (2015). Enhanced classification algorithms for the satellite image processing. Indian Journal of Science and Technology, 8(15).

4. Gharbia, R., El Baz, A. H., Hassanien, A. E., \& Tolba, M. F. (2014) Remote sensing image fusion approach based on Brovey and wavelets transforms. In Proceedings of the Fifth International Conference on Innovations in Bio-Inspired Computing and Applications IBICA 2014 (pp. 311-321). Springer International Publishing.

5. Blasch, Erik P., and Zheng Liu. "LANDSAT satellite image fusion metric assessment." Proceedings of the 2011 IEEE National Aerospace and Electronics Conference (NAECON). IEEE, 2011.

6. Al-Wassai, Firouz Abdullah, N. V. Kalyankar, and Ali A. Al-Zuky "The IHS transformations based image fusion." arXiv preprint arXiv:1107.4396 (2011).

7. Guo, Qing, et al. "Multispectral and panchromatic image fusion using a joint spatial domain and transform domain for improved DFRNT." Optik-International Journal for Light and Electron Optics 126.24 (2015): 5241-5248

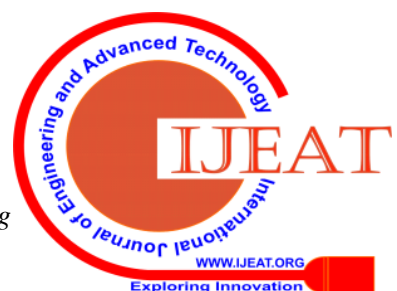




\section{A Segmentation Method of Fused Multispectral and Panchromatic Images using Quick Shift Algorithm and Brovey Transform}

8. Yang, Shuyuan, Min Wang, and Licheng Jiao. "Fusion of multispectral and panchromatic images based on support value transform and adaptive principal component analysis." Information Fusion 13.3 (2012): 177-184.

9. Salem, Muhammed, Abdelhameed F. Ibrahim, and Hesham A. Ali. "Automatic quick-shift method for color image segmentation." Computer Engineering \& Systems (ICCES), 2013 8th International Conference on. IEEE, 2013.

10. Bharathi, S., et al. "Performance analysis of segmentation techniques for land cover types using remote sensing images." 2012 Annual IEEE India Conference (INDICON). IEEE, 2012.

11. Wang, Zhijun, et al. "A comparative analysis of image fusion methods." IEEE transactions on geoscience and remote sensing 43.6 (2005):

12. 1391-402.

13. Rao, C. V., et al. "Satellite image fusion using fast discrete curvelet transforms." Advance Computing Conference (IACC), 2014 IEEE International. IEEE, 2014.

14. Ganesan, P., and V. Rajini. "Value based semi automatic segmentation of satellite images using HSV color space, histogram equalization and modified FCM clustering algorithm." Green Computing, Communication and Conservation of Energy (ICGCE), 2013 International Conference on. IEEE, 2013.

15. Li, C., \& Xu, D. (2009, May). Study on methods of fusion and classification using SPOT5 image of ZhongShan cemetery. In Urban Remote Sensing Event, 2009 Joint (pp. 1-5). IEEE.

16. Tian, H., Wang, P. G., \& Zheng, W. (2012, December). A new image fusion algorithm based on fractional wavelet transform. In Computer Science and Network Technology (ICCSNT), 2012 2nd International Conference on (pp. 2175-2178). IEEE.

17. Ulzurrun, E. I., Martín, C. G., \& Ruiz, J. M. (2016, June). Analysis of Land and marine resources by processing high resolution satellite images. In Information Systems and Technologies (CISTI), 2016 11th Iberian Conference on (pp. 1-4). IEEE.

18. Fan, J., Chen, T., \& Lu, S. (2015, December). Vegetation coverage detection from very high resolution satellite imagery. In Visual Communications and Image Processing (VCIP), 2015 (pp. 1-4). IEEE.

19. Mangai, U. G., Samanta, S., Das, S., Chowdhury, P. R., Varghese, K., \& Kalra, M. (2010, November). A hierarchical multi-classifier framework for landform segmentation using multi- spectral satellite images-a case study over the indian subcontinent. In Image and Video Technology (PSIVT), 2010 Fourth Pacific-Rim Symposium on (pp. 306-313). IEEE.

20. Tahoun, M., Shabayayek, A. E. R., \& Hassanien, A. E. (2014, May). Matching and co-registration of satellite images using local features. In Proceedings of the International Conference on Space Optical Systems and Applications, Kobe, Japan (Vol. 79).

21. Pandit, V. R., \& Bhiwani, R. J. (2015). Image fusion in remote sensing applications: A review. International journal of computer applications, 120(10).

22. Dalmiya, C. P., \& Dharun, V. S. (2015). A survey of registration techniques in remote sensing images. Indian Journal of Science and Technology, 8(26).

23. Mangai, U. G., Samanta, S., Das, S., Chowdhury, P. R., Varghese, K., \& Kalra, M. (2010, November). A hierarchical multi-classifier framework for landform segmentation using multi-spectral satellite images-a case study over the indian subcontinent. In Image and Video Technology (PSIVT), 2010 Fourth Pacific-Rim Symposium on (pp. 306-313). IEEE.

24. Parveen, R., Kulkarni, S., \& Mytri, V. D. (2016, March). Vegetation extraction based on spectral deviations using an IRS-1C LISS III image. In Wireless Communications, Signal Processing and Networking (WiSPNET), International Conference on (pp. 158-162). IEEE.

25. Sharma, M. (2016). A Review: Image Fusion Techniques and Applications. IJCSIT) International Journal of Computer Science and Information Technologies, 7(3), 1082-1085.

26. Parvatikar, M. V., \& Phadke, G. S. (2014). Comparative study of different image fusion techniques. International Journal of Scientific Engineering and Technology, 3(4), 375-379.

27. Sahu, D. K., \& Parsai, M. P. (2012). Different image fusion techniques-a critical review. International Journal of Modern Engineering Research (IJMER), 2(5), 4298-4301.

28. Kaur, V., \& Kaur, J. (2015). Comparison of Image Fusion Techniques: Spatial and Transform Domain based Techniques.

29. Bedi, S. S., \& Khandelwal, R. (2013). Comprehensive and comparative study of image fusion techniques. International Journal of Soft Computing and Engineering (IJSCE) ISSN, 3, 2231-2307.

30. [29] Morris, C., \& Rajesh, R. S. (2014). Survey of Spatial Domain Image fusion Techniques. International Journal of Advanced Research in Computer Science Engineering and Information Technology, 2(3).

31. Al-Wassai, F. A., Kalyankar, N. V., \& Al-Zuky, A. A. (2011). Arithmetic and Frequency Filtering Methods of Pixel-Based Image Fusion Techniques. arXiv preprint arXiv:1107.3348.

\section{APPENDIX}

\section{Source code}

clc

clear all

close all

warning off all;

$\% \%$ adding all sharpening toolboxes addpath(genpath([cd 'lbovey_toolbox'])); run([cd 'lother_toolboxlvlfeat-0.9.20\toolboxlvl_setup.m']); $\% \%$ input image getting $\mathrm{PAN}$ [filePAN,pathPAN]=uigetfile $\left(\left\{{ }^{\prime} * . j p g\right.\right.$ '.'*..png' $\left.\}\right)$; image_PAN=imread([pathPAN,filePAN]);

$\%$ figure,imshow(image_PAN);

$\%$ title('panachromic image');

$\%$ h = fspecial('motion', 50, 25);

$\%$ filteredimage_PAN $=$ imfilter(image_PAN, h);

$\%$ figure, imshow(image_PAN);

$\% \%$ input image getting multispectral

[file_multispectral,path_multispectral]=uigetfile( $\left.\left\{{ }^{\prime *} \cdot . j p g^{\prime} ;{ }^{\prime} * . p n g^{\prime}\right\}\right)$; image_MS=imread([path_multispectral,file_multispectral]);

figure,imshow(image_MS);

title('multispectral image');

$\% \%$ resampling

image_MS_rs=imresize(image_MS,size(image_PAN));

figure,imshow(image_MS);

title('resized multispectral image');

$\% \%$

if size(image_MS_rs,3) $<2$

warndlg('image must be color image....try with color image...'); else

$\% \%$ image co_registration

[optimizer, metric] = imregconfig('multimodal');

image_registered(:,:,1)=imregister(image_MS_rs(:,:,1),image_PAN $(:,:, 1)$,'affine',optimizer,metric);

image_registered(:,:,2)=imregister(image_MS_rs(:,:,2),image_PAN $(:,:, 1)$, 'affine',optimizer,metric);

image_registered(:,:,3)=imregister(image_MS_rs(:,:,3),image_PAN $(:,:, 1)$, 'affine',optimizer,metric);

figure,imshow(image_registered); title('co registered image');

$\% \%$

r=double(image_registered(:,:,1));

$\mathrm{g}=$ double(image_registered $(:,:, 2))$;

$\mathrm{b}=$ double(image_registered(:,:,3));

$\% \%$ for processing IHS transformation

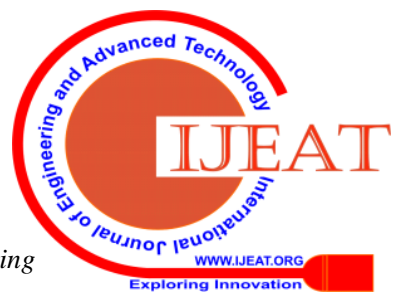


th $=\mathrm{acos}\left(\left(0.5^{*}((\mathrm{r}-\mathrm{g})+(\mathrm{r}-\mathrm{b}))\right) \cdot /\left(\left(\operatorname{sqrt}\left((\mathrm{r}-\mathrm{g}) \cdot{ }^{\wedge} 2+(\mathrm{r}-\mathrm{b}) \cdot *(\mathrm{~g}-\mathrm{b})\right)\right)+\mathrm{eps}\right)\right)$;

$\mathrm{H}=$ th;

$\mathrm{H}(\mathrm{b}>\mathrm{g})=2 * \mathrm{pi}-\mathrm{H}(\mathrm{b}>\mathrm{g})$;

image_MS_rs=imsharpen(image_MS_rs,'Radius',2,'Amount',4,'Thr

eshold',0.8);

$\mathrm{H}=\mathrm{H} /(2 * \mathrm{pi})$

$\mathrm{S}=1-3 \cdot *(\min (\min (\mathrm{r}, \mathrm{g}), \mathrm{b})) \cdot /(\mathrm{r}+\mathrm{g}+\mathrm{b}+\mathrm{eps})$;

$\mathrm{I}=($ uint $8(\mathrm{r})+$ uint $8(\mathrm{~g})+$ uint $8(\mathrm{~b})) / 3$;

hsi $=\operatorname{cat}(3, \mathrm{H}, \mathrm{S}, \mathrm{I})$;

$\%$ hsi=rgb2hsv(image_registered);

figure,imshow(hsi);

title('IHS image ');

$\mathrm{HE}=\mathrm{H} * 2 * \mathrm{pi}$;

HE=histeq(HE);

$\mathrm{HE}=\mathrm{HE} /(2 * \mathrm{pi})$;

$\mathrm{SE}=$ histeq $(\mathrm{S})$;

$\mathrm{IE}=$ histeq(I);

$\% \%$

figure,imshow(IE);

title('Intensity image histogram equalised ');

sharped $=$ solve_pansharp (uint8(I), uint8(IE));

new_intensity_image=uint8 $($ imresize $($ sharped,size $(\mathrm{I})))$;

$\% \%$ combining with IHS

IHS_recombined=cat(3,uint8(H),uint8(S),new_intensity_image);

$\% \%$ coverting to RGB from HSI image

Rgb_reconstructed=uint8(hsv2rgb(double(IHS_recombined)));

[optimizer, metric] = imregconfig('multimodal');

image_reconstruced(:,:,1)=imregister(image_MS_rs(:,:,1),Rgb_rec onstructed(:,:,1),'affine',optimizer,metric);

image_reconstruced(:,:,2)=imregister(image_MS_rs(:,:,2),Rgb_rec onstructed(:,:,2),'affine',optimizer,metric);

image_reconstruced(::,:3)=imregister(image_MS_rs(:,:,3),Rgb_rec onstructed(:,:,3),'affine',optimizer,metric);

figure,imshow(image_reconstruced); title('output fused RGB image ')

ratio $=0.5$;

kernelsize $=2$;

Iseg = vl_quickseg(image_reconstruced, ratio, kernelsize, 10);

figure,imshow(Iseg);

title('region based segmentation image');

$\% \%$

Iseg1=uint8(Iseg*255);

\section{AUTHOR PROFILE}

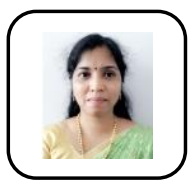

Dr. Vanmathi is an Associate Professor at School of Information Technology and Engineering at Vellore Institute of Technology, Vellore, India. received B.E degree in Computer Science from Madras University, M.Tech. in Information Technology from Sathyabama University and $\mathrm{PhD}$ in VIT Vellore. She has more than 12 years of experience in teaching and research. Her Research Interest includes Image Processing, Information Security, Machine Learning and IOT. 